\title{
Effect of the structured packing height on efficiency of freons mixture separation in a large-scale model of distillation column
}

\author{
Aleksandr Pavlenko*, Vladimir Zhukov, Nikolay Pecherkin, Aleksandr Nazarov, \\ Xingang Li, Mingyan Liu, Hong Sui and Hong Li \\ Kutateladze Institute of Thermophysics, Siberian Branch, Russian Academy of Sciences \\ 1 Lavrentyev Ave., Novosibirsk, 630090, Russia
}

\begin{abstract}
Results of experimental studies of heat-and-mass transfer and hydrodynamic processes at distillation on a regular packing are presented. The mixture of freons R114-R21 at the pressure of $0.3 \mathrm{MPa}$ was used as a working mixture. The mixture was separated on the Mellapak $350 Y$ structured packing with the diameter of $0.9 \mathrm{~m}$ under the conditions of complete reflux $(L / V=1)$ at different packing heights. A specially designed liquid distributor with a possibility to change the density and pattern of drip points was used to irrigate the packing. The experimental data on the efficiency of mixture separation (height of transfer unit HTU) and distribution of the local flow rate density over the column crosssection were compared. It is shown that an increase in the height of the structured packing from $2.1 \mathrm{~m}$ to $4.0 \mathrm{~m}$ leads to a significant decrease in the efficiency of mixture separation in the distillation column.
\end{abstract}

\section{Introduction}

Results of research and operation of industrial distillation columns with the regular packing have shown that often separation efficiency can decrease with an increase in their height and diameter, and this affects productivity and purity of the finished product. The fundamental phenomena responsible for a decrease in separation efficiency are the effects caused by transverse mal-distribution and longitudinal mixing. These effects relate to the vapor and liquid flow within the column, and they are presented by deviation of productivity and product purity from the calculated values at uniform distribution. The results published for laboratory samples of a small packing demonstrate an efficiency of about 20 or more theoretical stages per 1 meter of the packing height, while the efficiency of the best industrial samples is 5-6 theoretical stages per 1 meter of the height $[1,2]$. Setting the optimal structure of initial irrigation allows using the maximal packing height without significant deterioration of the efficiency of mixture separation caused by the negative factors in formation of large-scale mal-distribution of local mixture flows within the packing. The use of periodic packing irrigation, for example, allows destructing the stationary large-scale structures of uneven distribution of the local flows inside the packing

* Corresponding author: pavl@itp.nsc.ru 
and, accordingly, increasing separation efficiency of mixtures [3]. The purpose of this work is to investigate the effect of the regular packing height on the efficiency of Freon mixture separation, formation dynamics of large-scale mal-distribution of the local flow parameters over the packing cross-section along the column height for the given drip point pattern.

\section{Experimental setup and methods}

The experiments were carried out at the setup «Large Freon Column», whose detailed description is given in [2]. The mixture of freons R114-R21 at the pressure of $0.3 \mathrm{MPa}$ was used as the working mixture. This mixture was chosen to simulate the processes of cryogenic mixture separation. The mixture was separated on the regular Mellapak 350Y packing with the diameter of $0.9 \mathrm{~m}$ under the conditions of complete reflux $(L / V=1)$. The setups operated in the closed cycle. Two series of experiments were carried out with the packing height of 2.1 and $4.0 \mathrm{~m}$, respectively. A specially designed liquid distributor with the possibility to change the number and structure of drip points was used to irrigate the packing [3]. In these experiments, the rectangular drip point pattern with the density of $131 \mathrm{~m}^{-2}$ was applied. During the experiments, the efficiency of mixture separation (height of transfer unit $H T U$ ) was determined in the range of superficial vapor velocities of $0.017<K_{\mathrm{v}}<0.035 \mathrm{~m} / \mathrm{s}$ and the temperature field distributions in the packing cross-sections were registered at three levels along the column height. Distributions of the local liquid flow density, formed during the liquid film flow over the packing of various heights, were also obtained.

\section{Discussion}

Dependence of the mixture separation efficiency $(H T U)$ on superficial vapor velocity $\left(K_{v}\right)$ is shown in Fig. 1 for the column with the packing height of 4.0 and $2.1 \mathrm{~m}$. As it can be seen from the figure, the efficiency of mixture separation in the studied range of variations of superficial vapor velocity $0.017<K v<0.035 \mathrm{~m} / \mathrm{s}$ increases significantly with increasing $K_{v}$. An increase in the value of $K_{v}$ leads to a monotonic decrease in the height of transfer unit by the factor of 2.4-2.6, approximately. A significant decrease in the efficiency of mixture separation of the mixture at low values of superficial vapor velocities (and superficial liquid velocities, respectively) is caused by formation of local zones of deficient irrigation of the mass transfer surface inside the packing, and, as a result, its only partial wetting $[4,5]$. For equal values of $K_{v}$, the efficiency of mixture separation is considerably lower on the packing with the height of $4.0 \mathrm{~m}$. This result is explained by formation of large-scale maldistribution of the local mixture flow along the packing height, which causes a nonlinear effect (the so-called pinch effect [2]), which significantly reduces the efficiency of mixture separation. The main role in this negative influence is associated with formation of a large-scale maldistribution of the local liquid flow over the cross-section of the packing along its height, and, consequently, maldistribution of the local vapor flow.

The topograms of distribution of the local flow density of liquid flowing down from the lower edge of the packing are shown in Fig. 2, at its different heights and close values of $K_{v}$. As it follows from the analysis of experimental data presented in Fig. 2, distribution of the local liquid flow density at the outlet of the packing with the height of $4.0 \mathrm{~m}$ is slightly more uniform in comparison with distribution for the packing with the height of $2.1 \mathrm{~m}$. The values of standard deviation for the presented distributions are 11.5 and $14.5 \%$, respectively. At the outlet of the packing with the height of $2.1 \mathrm{~m}$, a higher density of the local liquid flow is observed at the packing periphery and in its central part. According to 


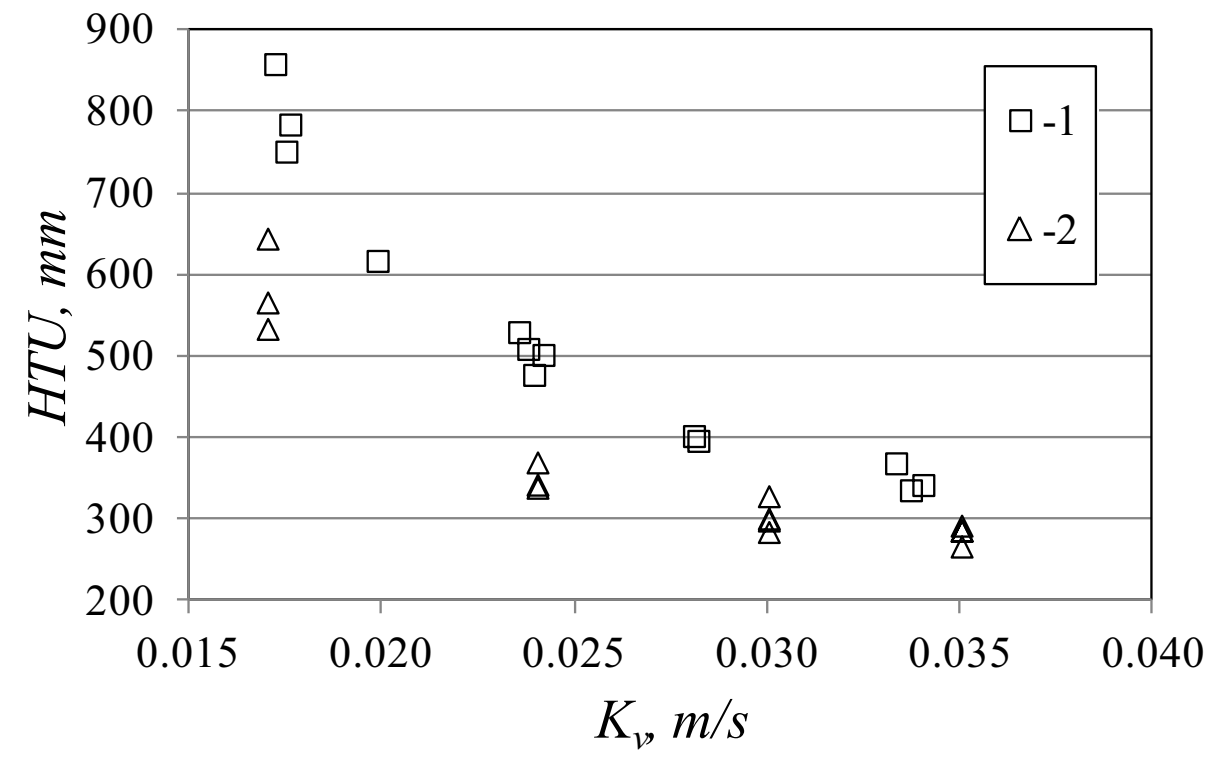

Fig. 1. Dependence $H T U\left(K_{\mathrm{v}}\right) ; 1-H=4.0 \mathrm{~m} ; 2-H=2.1$.

a)

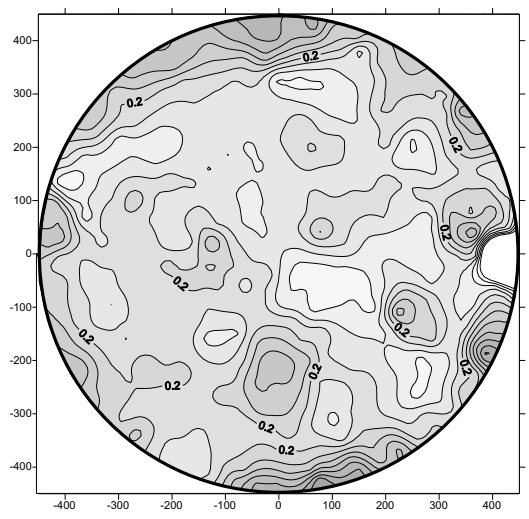

b)

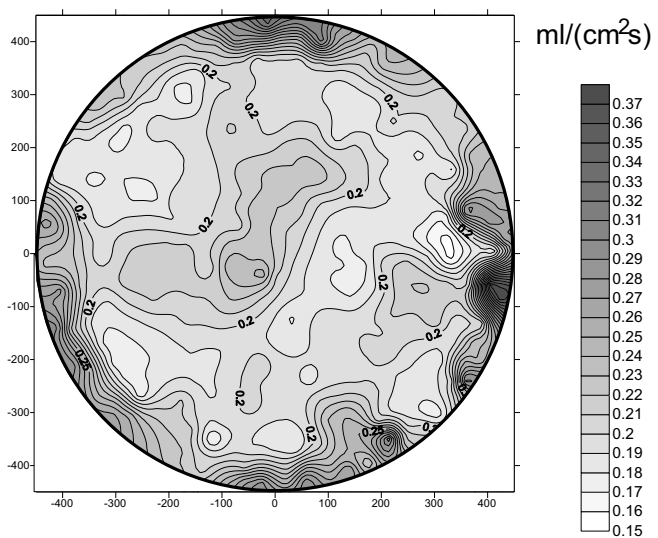

Fig. 2. Distribution of the local flow rate densty of liquid flowing from the lower edge of the packing. $K_{\mathrm{v}} \sim(0.027-0.030) \mathrm{m} / \mathrm{s} ; a-H=4.0 \mathrm{~m} ; b-H=2.1$.

the estimates obtained by the authors of [2] on the basis of the model of so-called divided column [6], the same degree of the local liquid flow mal-distribution over the packing cross-section has considerably greater negative effect on the height of transfer unit $H T U$ for the higher packing. This can explain the fact that for the close mal-distribution degrees determined in experiments for the local flow rates of liquid on the packing with different heights, the efficiency of mixture separation on the packing of $4.0 \mathrm{~m}$ height is much lower than that on the packing with the height of $2.1 \mathrm{~m}$. 


\section{Conclusions}

It is shown that an increase in the height of the structured packing Mellapak $350 Y$ from 2.1 to $4.0 \mathrm{~m}$ leads to a significant decrease in the efficiency of mixture separation in the distillation column with the diameter of $0.9 \mathrm{~m}$. Experimental data show close values of a degree of the local flow rate mal-distribution over the column cross-section for the packing of different heights. As it follows from the analysis, a significant increase in the height of transfer unit is caused by the fact that the same mal-distribution degrees of the local flow parameters for the packing of different heights have a more significant negative effect on the efficiency of mixture separation on the higher packing.

The obtained basic experimental data will be used for comparative analysis in further studies with varying density and structure of the drip points under the conditions of stationary and dynamically controlled laws of regular packing irrigation.

The study was performed at the IT SB RAS due to the grant of the Russian Science Foundation (project No. 14-49-00010).

\section{References}

1. V.S. Leontiev, S.I. Sidorov, Khim Prom. 82347 (2005)

2. A.N. Pavlenko, V.E. Zhukov, N.I. Pecherkin, V.Yu. Chekhovich, O.A. Volodin, A. Shilkin, C. Grossmann, AIChE J. 60, 690 (2014)

3. A.N. Pavlenko, V.E. Zhukov, N.I. Pecherkin, X. Li, H. Sui, J. Physics: Conference Series 754, 042012 (2016)

4. A.N. Pavlenko, X. Li, H. Li, X. Gao, O.A. Volodin, A.S. Surtaev, V.S Serdyukov, Techn. Phys. Lett. 41, 774 (2015)

5. A.N. Pavlenko, O.A. Volodin, A.S. Surtaev, Appl. Thermal Eng. 114, 1265 (2016)

6. M.J. Lockett, J.F. Billingham, Distillation and Absorption 2002 Proceedings (BadenBaden, Germany), (2002) 